\title{
FUNCIONAMIENTO Y ORDENACION DE LOS TRABAJOS DEL GOBIERNO
}

\author{
Por JOSE ANTONIO SANCHEZ MARISCAL \\ y VICTORIANO COLODRON GOMEZ
}

Sumario: I. INTROducción.-II. Instrucciones Reguladoras del Funcionamiento det GobieRno DESDE LA* LEY DE RÉGIMEN JURfDICO DE LA ADMINISTRación deI EsTADO: 1. Instrucción de servicio de 13 de diciembre de 1958. 2. Orden comunicada de 21 de noviembre de 1961. 3. Proyecto de Orden de febrero de 1963. 4. Escritos desde 1963 a 1970 . 5. Proyecto de instrucciones de 1970 y nota complementaria. 6. Nota de instrucciones de 1973. 7. Documento del año 1975. 8. Instrucciones de febrero de 1976.-III. La actual ORdENAción DaL Trabajo del Gobienno: 1. Intervención de los Departamentos ministeriales y Secretarías Generales Técnicas, 2. Comisiones interministeriales. 3. Disposiciones conjuntas. 4. Trabajos preparatorios, reuniones y trámites posteriores: la Comisión General de Subsecretarios: 4.1. Reparto previo de documentos. 4.2. Observaciones y contestaciones a las mismas. 4.3. Convocatoria de las reuniones. 4.4. Formación del orden del día. 4.5. Reuniones. 4.6. Actas. 4.7. Reflejo documental de los acuerdos. 4.8. Consideraciones. 5. Remisión a las Cortes de los proyectos de Ley y publicación de los acuerdos del Gobierno en el Boletin Oficial del Estado. 6. La Secretaria del Gobierno.-IV. ConCLUSIONES.

\section{Introducción}

El tema de la ordenación de los trabajos del Gobierno tiene un carácter adjetivo e instrumental, está poco tratado doctrinalmente e incluso su regulación, cuando no es consuetudinaria, suele plasmar en disposiciones de carácter interno no publicadas.

El carácter procedimental de la materia no disminuye, sin embargo, su trascendencia práctica en cuanto que un correcto funcionamiento del Gobierno condiciona en buena medida la coordinación política y administrativa, $\mathrm{y}$, en último término, la operatividad y buenos resultados de la política gubernamental. 
Si bien son relativamente abundantes los trabajos sobre la figura del primer ministro desde un punto de vista político y constitucional, sólo algunos presentan especifica atención a sus inmediatos órganos de apoyo. En cualquier caso, las escasas referencias a los mecanismos de funcionamiento del Gobierno suelen ser genéricas e insuficientes, debido sin duda a la discreción que rodea a sus trabajos y al difícil acceso a sus normas reguladoras. De ahí que la selección de estudios que se incluye al final de esta ponencia tenga un carácter básicamente orientativo.

Nuestra Administración no ha sido una excepción en la naturaleza reservada de las instrucciones ordenadoras de las tareas del Consejo de Ministros. Gracias a la colaboración de los funcionarios que, en la Secretaría General de la Presidencia del Gobierno, se ocupan diariamente de estas materias, ha sido posible no sólo conocer cada una de las instrucciones, acuerdos, notas o comunicaciones dictadas en los últimos veinte años, sino, lo que es más importante, la realidad de la preparación de las decisiones del Gobierno, que excede en ocasiones del contenido de la norma escrita.

Hay que tener en cuenta, de otro lado, que el ya apuntado carácter instrumental de este trabajo supone asimismo que difícilmente pueda llegarse a unas conclusiones finales sin definir previamente las grandes líneas de otras materias sustantivas objeto de otras ponencias. La forma en que se delimite el ejercicio de la potestad reglamentaria, el procedimiento que se perfile de elaboración de disposiciones generales y la configuración que se dé a los órganos directamente dependientes del presidente del Gobierno, por citar sólo las más significativas, son cuestiones que deben prejuzgar los criterios de ordenación del trabajo colectivo del Gobierno.

A la vista de estas limitaciones, parece lo más oportuno dedicar la parte central de la ponencia a describir el funcionamiento actual del Gobierno, lo que obliga a examinar las instrucciones que en orden a su regulación han ido dictándose desde la Ley de Régimen Jurídico de la Administración del Estado, ya que en buena parte de su contenido continúan estando en vigor. Además, el hecho de que nunca hayan sido publicadas, puede justi- 
ficar, aunque sea desde un punto de vista puramente documental, el reseñarlas con cierto detalle, aun a riesgo del carácter reiterativo del análisis.

Las necesarias referencias a otros sistemas comparados, así como las observaciones que determinados aspectos suscitan, se intercalarán a lo largo de la descripción, que se considera debe ser completada con el examen de los órganos que intervienen en el procedimiento, especialmente de la Secretaría del Gobierno.

II. Instrucciones reguladoras del funcionamiento del Gobierno desde la Ley de Régimen Jurídico de la Administración del Estado

No existe una norma jurídica, legal o reglamentaria, que regule la ordenación de los trabajos del Gobierno y la forma de preparar sus decisiones. Unicamente la Ley de Régimen Jurídico de la Administración del Estado y la Ley de Procedimiento Administrativo contienen algunos preceptos muy genéricos en la materia, los cuales han servido de punto de apoyo a una serie de instrucciones internas, dictadas en diferentes momentos a partir de la promulgación de los referidos textos legales y dirigidas a los órganos de la Administración que intervienen en el procedimiento de elaboración de dichas decisiones.

El artículo 12 de la Ley de Régimen Jurídico de la Administración del Estado alude al Secretariado de las Comisiones Delegadas del Gobierno, que "cuidará de la preparación de las reuniones del Consejo de Ministros y Comisiones Delegadas del Gobierno; de la distribución del orden del día y de cuantos datos e informes precisen los ministros para conocer los antecedentes de los asuntos sometidos a su deliberación; de levantar el acta de los acuerdos adoptados y velar por su ejecución; de cuidar de la inserción en el Boletín Oficial del Estado de los Decretos, Reglamentos y demás disposiciones generales del Gobierno y custodiar el archivo de sus minutas; registrar todas las disposiciones de carácter general y anotar sùs posteriores modificaciones o derogaciones».

Es curioso resaltar que pese a la denominación del órgano «Secretariado de las Comisiones Delegadas del Gobierno»-, se 
le encomiendan funciones respecto al Consejo de Ministros, y que en las sucesivas disposiciones que afectan a la estructura orgánica de la Presidencia del Gobierno, así como en las propias instrucciones internas antes referidas, se habla siempre de «Secretariado del Gobierno" y no de Secretariado de las Comisiones Delegadas.

Por lo que se refiere a la Ley de Procedimiento Administrativo, el capítulo primero de su título VI (arts. 129 a 132), relativo al procedimiento para la elaboración de disposiciones de carácter general, recoge una serie de formalidades que han de cumplimentarse a lo largo de este procedimiento especial, y en concreto, respecto a la cuestión que aquí nos interesa, el artículo 131 se limita a establecer la obligatoriedad del reparto previo de los proyectos que deban someterse a la aprobación del Consejo de Ministros o de las Comisiones Delegadas del Gobierno, los cuales «se remitirán, con ocho días de antelación, a los demás ministros convocados, con el objeto de que formulen las observaciones que estimen pertinentes. En casos de urgencia, apreciada por el propio Consejo de Ministros o Comisión Delegada, podrá abreviarse u omitirse este trámite». En cuanto a las leyes, el número 2 de dicho artículo añade que «el mismo procedimiento se observará para la aprobación por el Gobierno de los proyectos de ley que hayan de ser sometidos a las Cortes".

Aunque sea muy someramente, parece necesario examinar las sucesivas instrucciones internas que, en base a los preceptos legales citados, han tratado de ordenar en los últimos años los trabajos del Gobierno y que constituyen elemento de juicio esencial para explicar su funcionamiento actual.

1. El primer documento que debe reseñarse lo constituyen unas "instrucciones de servicio" del ministro subsecretario de la Presidencia del Gobierno a todos los ministros, de fecha $13 \mathrm{de}$. 'diciembre de 1958, cuyo contenido se centra casi exclusivamente en recordar la vigencia de ciertos preceptos de la Ley de Procedimiento Administrativo relativos a la tramitación de las disposiciones de carácter general, concretamente los siguientes:

- la iniciación de los proyectos de Ordenes, Decretos, Decretos-leyes y anteproyectos de Ley, deberá ir acompañada 
de los estudios e informes previos a que alude el artículo 129 ;

- será en todo caso inexcusable el informe de la Secretaria General Técnica o, en su defecto, de la Subsecretaría del Ministerio respectivo (art. 130, 1), indicándose que dicho informe del proyecto "versará especialmente sobre su adaptación a los planes generales de actuación del Departamento a que se refiere el artículo 19 de la Ley de Régimen Jurídico de la Administración del Estado";

- se requerirá la aprobación de la Presidencia del Gobierno (artículo 130,2 ) si las disposiciones se refieren a «estructura orgánica, métodos de trabajo burocrático, procedimiento y personal de la Administración Pública";

- el proyecto deberá someterse a dictamen del órgano u ór-ganos consultivos determinados por las disposiciones vigentes (art. 130, 3), conteniéndose una especial referencia al Consejo de Estado, cuyo dictamen se advierte que tiene carácter preceptivo en los casos que se enumeran en la entonces última. Resolución del Alto Cuerpo Consultivo sobre el tema (de 25 de septiembre de 1958);

- la nueva disposición deberá acompañarse de una tabla de vigencias y aquélla consignará expresamente las anteriores disposiciones que han de quedar total o parcialmente derogadas (art. 129, 3);

- todo proyecto que pretenda crear un nuevo órgano deberá cumplir los requisitos exigidos por el artículo $3 .^{\circ}$ de la Ley de Procedimiento Administrativo: determinación del Departamento en que se integra, supresión o restricción de las competencias de los que supongan duplicación y elaboración del previo estudio económico del coste de su funcionamiento y del rendimiento o utilidad de sus servicios.

La única parte significativa de esta «instrucción de servicio» es su último punto, que establece lo siguiente: «Los proyectos que deban someterse a la aprobación del Consejo de Ministros o de sus Comisiones Delegadas, han de remitirse con ocho dias de 
antelación, por el Secretariado del Gobierno, a los ministros convocados, a cuyo efecto los respectivos Departamentos deberán remitir a dicho Secretariado sus proyectos antes del indicado plazo (art. 131 de la Ley de Procedimiento Administrativo.n)

Como fácilmente se advierte, y quizá en un primer intento de ordenar el trabajo del Gobierno por lo que a las disposiciones generales se refiere, este punto hace una interpretación forzada del artículo 131 de la Ley de Procedimiento Administrativo al exigir que el reparto de los proyectos se realice a través del Secretariado del Gobierno, obligando con ello a ampliar el plazo establecido en dicho precepto legal.

Si comparamos esta primera instrucción con las que sucesivamente se dictan, cabe advertir, junto a la insuficiencia de su contenido, el tono poco imperativo de su introducción $(« \ldots$ si V. E. lo estima oportuno se dé traslado a las autoridades y funcionarios dependientes de $\mathbf{V}$. $E$. de las siguientes instrucciones de servicio..."), que contrasta con la rigidez del punto relativo al reparto de proyectos (todos se distribuirán a través y por el Secretariado del Gobiernol, sucesivamente reiterado en posteriores instrucciones, incluso en términos más flexibles, lo que parece poner de relieve su sistemático incumplimiento.

2. Con fecha 21 de noviembre de 1961, y como resultado de los trabajos realizados por una Comisión Interministerial creada el año anterior e integrada por representantes de todos los Departamentos ministeriales, se dicta una Orden comunicada de la Presidencia del Gobierno por la que se aprueban las aInstrucciones sobre normalización de documentos y expedientes que se elevan al Consejo de Ministros y Comisiones Delegadas del Gobierno y su tramitación".

Estas Instrucciones habrian de entrar en vigor el 1 de enero de 1962, prohibiéndose al Secretariado del Gobierno dar trámite, a partir de esta fecha, a la documentación que no se sujetara exactamente a las mismas.

A diferencia de la instrucción reseñada de 1958, las normas que ahora se examinan constituyen el primer intento de ordenar con minuciosidad las distintas fases del trabajo dèl Gobierno. Además de aprobar cuatro modelos normalizados de impresos para todos los asuntos que se eleven al Consejo de Ministros y 
Comisiones Delegadas del Gobierno, las normas -que contienen 23 puntos, agrupados en cinco epígrafes- regulan fundamentalmente los aspectos que seguidamente se señalan.

Los asuntos objeto de consideración del Gobierno se clasifican en dos grandes categorías: disposiciones de carácter general y expedientes", englobando bajo esta última y genérica denominación el resto de los asuntos cuya aprobación corresponde al Consejo de Ministros.

Respecto a los «expedientes», se presta especial atención a las cuestiones formales, estableciendo que los mismos se elevarán únicamente en «extracto», cuyo contenido (denominación, propuesta, exposición y dictámenes y trámites preceptivos), datos de identificación y garantías formales de autenticidad, son regulados con cierta minuciosidad. Estos "extractos" deben remitirse al Secretariado del Gobierno con una antelación mínima de cuarenta y ocho horas a la fecha de la reunión del Consejo de Ministros.

En cuanto a las disposiciones de carácter general, con independencia de alguna referencia formal (modelo normalizado en que deben transcribirse y diligencia de elevación del proyecto), la Instrucción se centra en los plazos y forma de reparto previo. Como regla general se establece el plazo de ocho días de antelación mínima, permitiendo que dicho reparto se haga a través del Secretariado del Gobierno o directamente por el Ministerio proponente al resto de los Departamentos. En este último supuesto se exige que, al pedir la inclusión del asunto en el orden del día, se haga constar que el reparto ha sido realizado y la fecha en que se efectuó. El incumplimiento del plazo mínimo de reparto no determina, según las Instrucciones, la exclusión del proyecto del orden del día, sino que se hace constar en el mismo tal circunstancia a fin de que el Gobierno decida previamente sobre el carácter de urgencia del asunto en cuestión para entrar o no a conocer sobre el fondo.

Otro aspecto tratado con minuciosidad en las Instrucciones es el relativo a la elaboración del orden del día de las reuniones del Gobierno.

Cada titular de Departamento ministerial prepara el índice de asuntos que decide someter a la consideración del Gobierno, 
remitiéndolo al secretario del Gobierno al menos con cuarenta y ocho horas de antelación a la reunión. En base a estos índices, el Secretariado del Gobierno elabora el orden del día y lo distribuye a los ministros la víspera de la sesión.

El orden del día consta de tantos apartados como ministros actuantes y por el turno de intervención previstos para los mismos. Cada uno de estos apartados tiene idéntica estructura, que se ajusta a la siguiente clasificación:

1. Disposiciones generales.

1.1. Anteproyectos de Ley.

1.2. Proyectos de Decreto-ley.

1.3. Proyectos de Decreto.

1.4. Proyectos de Orden.

2. Otras disposiciones.

2.1. Anteproyectos de Ley.

2.2. Proyectos de Decreto-ley.

2.3. Proyectos de Decreto.

2.4. Proyectos de Orden.

3. Informes.

4. Mociones y propuestas.

5. Expedientes.

Por último, respecto a los trámites posteriores a las reuniones, se regula el diligenciado y devolución de la documentación remitida, que realizará el Secretariado del Gobierno dentro de los cuatro días hábiles siguientes a la fecha de la reunión, así como los supuestos de modificaciones introducidas en las propuestas elevadas. En estos casos los ministros proponentes deben remitir al ministro subsecretario de la Presidencia del Gobierno, y en el plazo de tres días hábiles, Nota en que se recojan tales modificaciones.

El análisis del contenido de estas instrucciones permite afirmar que constituyen uno de los pocos intentos de ordenar con cierta ambición y rigor el funcionamiento del Gobierno. De hecho, buena parte de su contenido ha conservado su vigencia hasta la 
fecha. No obstante, conviene subrayar algunas de sus más significativas limitaciones:

- no se indica el órgano que aprueba el orden del día, lo que quizá no es de extrañar dada la confusión existente en estos momentos entre la Jefatura del Estado y la Presidencia del Gobierno;

- se rehúye especificar el turno de intervención de los ministros en las reuniones, posiblemente por el carácter más bien procedimental y administrativo de la instrucción;

- no existe una sola referencia a las actas de los Consejos, e incluso la alusión que se hace a las modificaciones que se introducen en las propuestas da a entender que aquéllas no se confeccionan en base a unas notas del ministro secretario del Consejo, como resulta normal en el funcionamiento de los órganos colegiados, sino atendiendo a las notas que cada miembro del Gobierno confecciona en los asuntos de su competencia.

3. En febrero de 1963 se eleva al ministro subsecretario de la Presidencia del Gobierno, posiblemente por la Secretaría General Técnica, un proyecto de Orden comunicada que no llegó a firmarse, recayendo el acuerdo de que se haga una gestión privada con las Secretarias Generales Técnicas.

$\mathrm{Su}$ contenido se centra simplemente en recordar la estricta observancia del plazo mínimo de ocho días prescrito en el artículo 131 de la Ley de Procedimiento Administrativo para el reparto de los proyectos de disposiciones generales, excepto en los casos de urgencia, que será apreciada por el propio Consejo de Ministros.

La única novedad que este proyecto intentaba introducir consistía en que serian excluidos automáticamente del orden del. día de las reuniones los demás asuntos que sin tener el carácter de urgentes fueran repartidos fuera de plazo.

La conclusión que parece poder sacarse de esta propuesta es que los plazos a que alude no son generalmente cumplidos y que se intentan reducir más bien verbalmente los supuestos de asuntos calificados de urgentes. 
4. Desde 1963 hasta 1970 solamente tres escritos sobre la materia que se limitan a recordar algunos de los aspectos de las instrucciones ya comentadas.

El primero de ellos, de fecha 24 de noviembre de 1965, lo dirige el ministro subsecretario de la Presidencia del Gobierno, y señala la necesidad de que el índice de asuntos que los Departamentos deben tratar en el Consejo sean remitidos al Secretariado del Gobierno con cuarenta y ocho horas de antelación, comprometiéndose la Presidencia a repartir el orden del día con veinticuatro horas de anticipación.

El siguiente está firmado por el ministro secretario del Consejo de Ministros, con fecha 12 de febrero de 1968, e insiste en el mismo tema, si bien fija como tope las doce horas del miércoles respectivo.

Por último, el 27 de febrero de 1970, el vicepresidente del Gobierno dirige a los Ministros un escrito cuyo contenido coincide exactamente con el anterior.

Como se advierte, el reiterado y escaso contenido de estos escritos, cuya reseña obedece fundamentalmente al deseo de dejar constancia de toda la documentación sobre el tema, parece indicar que los mismos se producen en momentos próximos a la entrada de nuevos miembros en el Gobierno.

5. En el año 1970 se produce un intento desde la Presidencia del Gobierno de renovar y sustituir las instrucciones de funcionamiento del Gobierno. En esta época coinciden una serie de circunstancias tanto políticas como de carácter tẹ́cnico que favorecen dicho intento. De un lado, existe un Gobierno más homogéneo que los anteriores que parece facilitar una ondenación más rigurosa de sus trabajos, lo que posiblemente se une a un deseo de concentrar mayor poder en los órganos directamente dependientes del vicepresidente del Gobierno. Por otra parte, se empieza a sentir la necesidad de que el Gobierno, cualquiera que sea su composición, debe desconcentrar sus atribuciones, dejando de decidir sobre determinados asuntos $\mathrm{y}$, sobre todo, debe alterar sus métodos de trabajo: se tiene el convencimiento de que el Consejo de Ministros no debe deliberar sobre textos jurídicos acabados, sino adoptar grandes decisiones en los distintos cam- 
pos de la política nacional, evitando que gran parte de sus sesiones se dediquen posiblemente por la formación profesional y técnica de la mayoría de sus miembros, a formular observaciones respecto a la perfección formal de un proyecto determinado.

Sin embargo, como se verá más adelante, este intento sólo llega a ponerse en vigor muy parcialmente $\mathrm{y}$ en aspectos sobre todo formales. .

En los primeros meses de 1970 los servicios de la Secretaría General Técnica de la Presidencia del Gobierno elaboraron un proyecto de instrucciones que fue distribuido a todos los Departamentos a fin de contar con el parecer de los mismos antes de su aplicación.

$\mathrm{Su}$ contenido era bastante amplio y por primera vez se trataba de recoger en un solo documento las distintas etapas des trabajo del Gobierno, desde las reuniones preparatorias de los. Consejos de Ministros hasta la inserción de sus decisiones en el Boletin Ofictal del Estado.

El proyecto aborda aspectos de trabajo del Gobierno nunca tratados en instrucciones anteriores, como, por ejemplo, el orden de actuación de los ministros en las reuniones o toda la regulación relativa a la redacción de las actas, aspectos todos ellos que inciden en una consideración más política del tema.

De otro lado, es también significativo el intento de modificar el método de trabajo del Gobierno, que deberia deliberar por regla general sobre distintas opciones o alternativas en cada caso («asuntos para deliberación»), encargando a los Ministerios interesados la elaboración del proyecto de disposición o acuerdo que sería objeto posteriormente de la definitiva aprobación («asuntos para decisión»).

Sin embargo, las innovaciones del texto, posiblemente contestadas por los Departamentos en las reuniones tenidas al efecto, aconsejaron la puesta en vigor parcial del mismo. De hecho, con fecha 6 de octubre de este mismo año, el vicepresidente del Gobierno remitió a todos los ministros una «Nota» que recogía los puntos de las instrucciones que deberían aplicarse a partir del Consejo de Ministros del día 23.

La “Nota» constituye en sí misma unas instrucciones mucho 
más modestas y en cuya regulación conviene destacar algunos puntos.

Los proyectos deben repartirse con la antelación suficiente para ser objeto de un detenido estudio. Por lo tanto, el plazo minimo de ocho días de reparto no garantiza la inclusión automática: en el orden del día, ya que tal inclusión se producirá cuando su grado de elaboración permita prever que puede ser objeto de aprobación. Tanto es así que se exige que el Ministerio proponente, al distribuir el asunto, señale la fecha de la reunión en que estima debe ser sometido a la consideración del Gobierno. De otro lado, el reparto se realizará inexcusablemente a través del Secretariado del Gobierno.

Las observaciones de los demás Ministerios proponiendo modificaciones a los proyectos son objeto por primera vez de cierta atención. Su objeto debe ser reflejar el parecer del Departamento que las formula desde el punto de vista de su competencia específica y de acuerdo con la política general del Gobierno, evitándose el envío de escritos procedentes de distintos Centros directivos de un mismo Departamento. Se remiten directamente al Ministerio proponente, con cuatro días de antelación a la reunión, enviándose un duplicado a la Presidencia del Gobierno. A la vista de las observaciones, el proponente fija su postura definitiva, bien contestándolas o aceptándolas, bien elaborando un nuevo texto, documentación que asimismo ha de enviarse a la Presidencia del Gobierno.

También son objeto de consideración los proyectos conjuntos, es decir, los que afectan a la competencia de varios Departamentos, si bien la regulación se queda en un plano puramente formal.

La "Nota" mantiene la posibilidad de que se eleven «asuntos para deliberación", que son remitidos a todos los Departamentos con setenta y dos horas de antelación, y respecto a los cuales se ha perdido el carácter que quiso darle las instrucciones.

En la formación del orden del día se contempla, por un lado, su estructura, que es sensiblemente análoga a la que estaba en vigor, si bien se distinguen los asuntos para decisión de los de deliberación e información; y de otro, su elaboración y aprobación. El orden del día continúa elaborándose en base a los ín- 
dices enviados por los Departamentos, aunque se establece el requisito de que los proyectos de disposición hayan obtenido la conformidad de principio de los Ministerios interesados, de tal modo que pueda preverse su aprobación. Antes de distribuirse a los Departamentos, el Secretariado del Gobierno lo somete a la aprobación del vicepresidente del Gobierno.

En febrero de 1971 se sircula una adición sobre el reparto de los Convenios y Acuerdos Internacionales como consecuencia de las dificultades puestas de relieve por el Ministerio competente y cuyo contenido tiende a permitir el reparto en extracto de dichos asuntos y a dar mayor facilidad para su inclusión entre los temas calificados de urgentes.

Por último, a finales de este año se produce un escrito del vicepresidente del Gobierno a todos los ministros recordando la vigencia de la "Nota" y poniéndose especial énfasis en los plazos de envio de documentos y observaciones.

6. En junio de 1973 el ministro subsecretario de la Presidencia del Gobierno envía a todos los ministros una nueva nota de instrucciones, que es prácticamente reproducción de la deI año 1970, con las modificaciones necesarias derivadas del nombramiento de presidente del Gobierno.

Dentro de la regulación de "otras disposiciones" se alude expresamente por primera vez a la necesidad de que las propuestas de nombramiento sean consultadas en despacho con el presidente del Gobierno.

7. En el año 1975 se elaboró un nuevo documentọ, que tuvo carácter reservado y que si bien no consta su distribución y aplicación efectiva, su contenido, sin duda, informa los trabajos del Gobierno y sus verdaderos destinatarios son los ministros más que los órganos administrativos correspondientes.

Aunque su preámbulo señala como finalidades de estas normas las de asegurar el ejercicio de las facultades de dirección y coordinación del presidente del Gobierno, evitar que se sometan al Gobierno proyectos de disposiciones no suficientemente elaborados y descargar de su deliberación los asuntos de trámite o de escasa significación política, lo cierto es que no se alcanza 
el verdadero sentido de las normas sin considerar el momento político en que se producen.

Estamos en los últimos meses del anterior régimen en los que el Gobierno duplica sus reuniones a través de lo que en los medios de información vienen a denominarse «Consejos deliberantes» $\mathrm{y}$ «Consejos decisorios». Estos últimos son los jurídicamente válidos y se celebran bajo la presidencia del Jefe del Estado, mientras que los «deliberantes», presididos por el presidente del Gobierno, formalmente son reuniones simplemente preparatorias, pero en la práctica son auténticos Consejos donde se salvan la mayor parte de las discrepancias que no se desea que se reproduzcan ante el Jefe del Estado.

De ahí que las normas otorguen una serie de facultades al presidente del Gobiermo (por lo demás no excepcionales desde un punto de vista formall que no son sino cautelas para garantizar la rigidez del desarrollo de las reuniones decisorias. Así, el presidente ordena el reparto de todos los textos, determina la reunión en que serán examinados, es informado con carácter previo y reservado del grado de conformidad y discrepancias existentes, decide qué informes deben ser objeto de intervención oral e incluso determina los Ministros que especificamente deben intervenir en cada sesión.

En este mismo sentido se establece un orden del día abreviado («orden del día rojo») que sólo contiene los asuntos que deben ser objeto de deliberación, considerándose aprobados todos los demás asuntos contenidos en el orden del día elaborado inicialmente («orden del día negro»).

8. Unas nuevas instrucciones, otra vez de carácter básicamente administrativo, son aprobadas en febrero de 1976, que únicamente son aplicadas durante unos meses, hasta la entrada en vigor de las normas vigentes, que serán consideradas al tratar del funcionamiento actual del Gobierno.

Si hay algo destacable en ellas, ya que vienen a ser en cierto modo una reproducción de anteriores normas, es la intervención de la Dirección General de Relaciones Institucionales, Centro directivo en el que está encuadrado en ese momento el Secretariado del Gobierno, y el hecho de que se prevean reuniones de secretarios generales técnicos, que, presididas por el ministro o el 
subsecretario de la Presidencia, tienen por objeto armonizar disparidades de criterios en torno a los proyectos de disposiciones para contribuir a su elaboración definitiva.

Estas reuniones, que de hecho sólo se celebrarán en contadas ocasiones $\mathrm{y}$ en torno a cuestiones concretas de cierta complejidad, pueden considerarse precedentes de la Comisión General de Subsecretarios que más adelante se examinará.

\section{La actual ordenación del trabajo del Gobierno}

La descripción del funcionamiento actual del Gobierno exige no sólo considerar las instrucciones vigentes emanadas del propio Consejo de Ministros, contrastadas con la práctica diaria, sino también prestar alguna atención a las Comisiones Interministeriales que tienen por objetivo la elaboración de borradores de proyectos de disposiciones, hacer una especial referencia a las «disposiciones conjuntas», cuyo procedimiento de elaboración presenta algunoš matices respecto al común, $\mathrm{y}$, finalmente, aludir al procedimiento regulador del envio de documentos a las Cortes en cuanto afecte a la última fase de aprobación y promulgación de disposiciones en las que éstas intervienen.

Simultáneamente se harán las imprescindibles referencias orgánicas para completar el esquema procedimental. En este sentido se aludirá a las Secretarias Generales Técnicas de los distintos. Departamentos, al encuadre del Secretariado del Gobierno en la organización de la Presidencia del Gobierno, a la Comisión General de Subsecretarios, etc.

Por último, necesidades de sistemática aconsejan acompañar esta descripción de algunos comentarios críticos y, con las limitaciones señaladas en la Introducción, de referencias a otros. sistemas comparados.

1. Las decisiones que adopta el Gobiemo suelen nacer en los distintos Departamentos y experimentan en éstos un previo proceso de elaboración, que no es objeto de atención en las instrucciones reguladoras del funcionamiento de aquél, centradas en la tramitación de los asuntos desde que se elevan a Consejo. 
Este modo de actuar, el que la iniciativa de la mayoria de los proyectos parta de los distintos Departamentos - y dentro de ellos de sus diferentes Centros directivos-, contrasta con otros sistemas de organización del trabajo colectivo del Gobierno, como, por ejemplo, el francés, que descansa en la programación previa de objetivos del Gobierno para un periodo determinado, lo que supone en la práctica la fijación de un calendario al que se ajustan los Departamentos en la preparación de las disposiciones que van a ser objeto de deliberación, tanto en las distintas fases de su elaboración como en la fecha en que se prevé su inclusión en el orden del día.

Siendo ello así, conviene dedicar unas líneas a describir la apuntada fase previa. Los proyectos de disposiciones se inician en el Centro directivo competente en la materia o excepcionalmente en las propias Secretarias Generales Técnicas. De su tramitación se encargan normalmente las Subsecretarías, que recaban los informes y dictámenes preceptivos. Entre éstos está el de la Secretaria General Técnica a que se refiere el articulo 130, 1 de la Ley de Procedimiento Administrativo.

La intervención de las Secretarias Generales Técnicas no se limita a esto, sino que generalmente elaboran también los informes a las disposiciones preparadas por otros Ministerios en el reparto previo a la elevación a Consejo - tarea que en algunos Ministerios realizan los servicios de la Subsecretaría o el propio Gabinete del Ministro-. Son los informes que se envían a los Ministerios proponentes cuando hay observaciones que formular y que incluso llevan los ministros en la "cartera de Consejo".

Teóricamente estos informes deben recoger las distintas opiniones que puedan producirse dentro de un Departamento respecto a las disposiciones elaboradas por los demás. Sin embargo, todavia existen Ministerios en los que la Secretaría General Técnica no logra "centralizar" estas opiniones, circulándose diversos escritos de observaciones, lo que dificulta, siquiera en pequeña medida; la preparación de las tareas del Gobierno.

Respecto a su contenido, merece destacarse que su generalización en todos los Departamentos en los últimos diez o quince años ha contribuido positivamente al perfeccionamiento técnico 
y juridico de los textos publicados. En contrapartida, debe ponerse de manifiesto que la parte de observaciones de estos informes en contadas ocasiones inciden en materia de oportunidad, lo que ha ocasionado que los ministros requieran a veces otras Notas y el que los informes que se circulan sobre un mismo proyecto vengan a ser en gran medida reiterativos. Esto ha originado, junto a otras causas, y sobre todo en anteriores Gobiernos "tecnocráticos", que en ocasiones la deliberación del Consejo pudiera perderse en consideraciones de técnica jurídica y consulta de textos legales.

En una reconsideración postconstitucional de la organización de los trabajos del Gobierno, parecería más adecuado y conveniente determinar un órgano, posiblemente encuadrado en la Presidencia del Gobierno, dedicado a velar por la perfección formal de las disposiciones -al igual que el Ministerio de $\mathrm{Ha}$ cienda lo hace en exclusiva respecto a su repercusión en el gasto público-, mientras que los informes de los demás Ministerios se dedicarian a analizar fundamentalmente la disposición desde su incidencia en las competencias que éstos tienen atribuidas y su encaje en el programa general del Gobierno, sin perjuicio de que las observaciones técnicas y jurídicas que consideren oportuno hacer fueran atratadas" y coordinadas con posterioridad en la Secretaría General del Gobierno.

2. Además del procedimiento señalado en el punto anterior, algunos proyectos de disposiciones son elaborados, al menos en su primera redacción, en las denominadas Comisiones Interministeriales.

Suele ser utilizada esta formula de elaboración en aquellos supuestos en que la norma incide en el ámbito de competencia de varios Departamentos.

La constitución de estas Comisiones puede decidirse a iniciativa del Departamento con competencia preponderante sobre la. materia a regular o porque asi lo prevé expresamente una disposición anterior, pero en todo caso desde el inicio del proceso de la elaboración de la norma, o, por el contrario, en otras ocasiones, redactado un proyecto y elevado al Consejo de Ministros, es el propio Gobierno, a la vista de la naturaleza de las observa- 
ciones formuladas, el que decide por razones de oportunidad la creación de la Comisión.

Desde el punto de vista del objeto de esta ponencia, deben considerarse como Comisiones Interministeriales todas aquellas que tienen por finalidad la redacción de disposiciones generales, estén o no formalizadas jurídicamente.

Las primeras entran dentro del ámbito del Decreto de 7 de julio de 1965 (desarrollado por Onden de 24 de mayo de 1969) y se constituyen por wacuerdo del Consejo de Ministros o de las Comisiones Delegadas del Gobierno o por resolución de la Presidencia del Gobierno».

Estas dos normas intentan controlar su creación y la marcha de sus trabajos a través de un Registro encuadrado en el Secretariado del Gobierno, si bien en la práctica este control ha sido poco eficaz debido al incumplimiento de las obligaciones que las normas imponen a las propias Comisiones.

Al lado de éstas, frecuentemente surgen en nuestra Administración grupos de trabajo, comisiones ad hoc o de expertos, etc., integradas por representantes de los Departamentos a muy diferente nivel, según los casos, con una duración temporal muy limitada y ninguna o escasa formalización. Sus trabajos sustituyen con ventaja por su mayor agilidad y flexibilidad al procedimiento ordinario de cruce de Notas formales de observaciones y contestaciones.

Pensamos que esta forma de preparación de decisiones del Gobjerno deberia generalizarse para aquellos supuestos, que siempre serán muy numerosos, en que están afectadas las competencias de diversos Ministerios, sin que se aprecie ventaja sustancial en la excesiva formalización y rigidez de su creación y funcionamiento. Se trataria, al igual que ocurre en otras Administraciones, de que se establezcan los supuestos en los que es conveniente que surjan estas reuniones interministeriales, previendo la presencia en todas ellas de un representante, del nivel adecuado, de la Secretaría del Gobierno que, además de ofrecer una asistencia técnica, seguiria muy de cerca sus trabajos e impulsaría su marcha en caso necesario.

En Alemania, por ejemplo, es obligatoria la deliberación de ministros interesados o sus representantes en aquellos casos en 
que existen diferencias de opinión, como requisito para someter el tema al Gobierno Federal. Algo parecido ocurre en el sistema francés, en donde, con independencia de las posibles reuniones de ministros interesados, el Presidente del Consejo puede provocar, normalmente para la articulación de textos sobre los que ya ha recaído acuerdo de principio, reuniones de representantes de los Ministerios afectados a las que asiste un representante del secretario general del Gobierno.

3. Además de la mayor parte de los proyectos de disposiciones redactados por Comisiones Interministeriales, existen otros que, por afectar al ámbito de competencias de varios Departamentos, revisten también la forma de "disposiciones conjuntas".

La regulación legal de las mismas está contenida en el artículo 24, 2, de la Ley de Régimen Jurídico de la Administración del Estado, que establece que los Decretos, cuando afectan a varios Ministerios, se dictarán a propuesta de los ministros interesados y serán refrendados por el presidente del Gobierno o el ministro subsecretario de la Presidencia (actualmente ministro de la Presidencia).

Con independencia del procedimiento práctico utilizado en cada caso para lograr el acuerdo de los ministros interesados, la peculiaridad formal de estos Decretos reside en el requisito de que el «elévese a Consejo" sea firmado por todos los ministros proponentes.

El citado artículo 24, 2, de la Ley de Régimen Jurídico de la Administración del Estado no ha sido nunca objeto de desarrollo, por lo que la tramitación de estas disposiciones no ha sido en todo momento idéntica, sino que ha obedecido a distintos criterios prácticos. Siempre, no obstante, se han incluido en el orden del día del Consejo como asuntos de Presidencia, han sido refrendados por el ministro de la Presidencia y no por el presidente del Gobierno, y para su inclusión en el orden del día se comprueba simplemente la conformidad de los Ministerios interesados cuando la propuesta no ha sido convenientemente formalizada según lo indicado anteriormente.

Si en la futura regulación de la potestad reglamentaria del Gobierno se aceptase, como parece que seria de desear, el "refrendo múltiple" de las disposiciones emanadas del Consejo de $\mathrm{Mi}$ - 
nistros que afecten a más de un Departamento, la especialidad de las "disposiciones conjuntas" debería desaparecer.

Cuestión distinta seria que se previeran, supuesto asimismo aconsejable, Decretos del presidente del Gobierno no deliberados en Consejo de Ministros -equivalentes a los «Decretos ordinarios" franceses-; que en gran parte recogerian las materias actualmente objeto de las "Ordenes conjuntas" (art. 25, 2, de la Ley de Régimen Jurídico de la Administración del Estado).

4. La organización actual de los trabajos del Gobierno tiene su origen en el Acuerdo del Consejo de Ministros de 1 de octubre de 1976 por el que se constituye la Comisión General de Subsecretarios, poniendo en práctica lo ya previsto en el artículo $8 .^{\circ}$ de la Ley de Régimen Jurídico de la Administración del Estado.

Los cometidos que formalmente se le encomiendan son los siguientes:

- proceder al examen de aquellos asuntos que el Consejo de Ministros le encomiende, cuando haya acordado posponerlos para ulterior deliberación.

- instrumentar las medidas técnicas y administrativas necesarias para una más exacta y puntual aplicación de las decisiones del Gobierno que afectan a varios Departamentos ministeriales y velar por la ejecución coordinada de las mismas.

- conocer de los asuntos propios del Gabinete que el presidente del Gobierno, a iniciativa propia o de los ministros interesados, considere oportuno que sean estudiados previamente por la Comisión General de Subsecretarios.

- asistir a la Presidencia del Gobierno en el ejercicio de sus funciones de coordinación administrativa y demás atribuciones específicas de alcance interministerial.

- desempeñar cualesquiera otros cometidos análogos que le confíen el Consejo de Ministros o el presidente del Gobierno.

Si bien la Comisión se reunió inicialmente para abordar con carácter esporádico temas de la competencia de la Presidencia del Gobierno que afectaban a la generalidad de los Departamentos, muy pronto se dio periodicidad a sus reuniones con el 
objeto casi exclusivo de examinar con carácter previo los asuntos que han de ser sometidos a la consideración del Consejo de Ministros, por lo que en la actualidad su actuación es elemento esencial en la ordenación de los trabajos del Gobierno.

La Presidencia de la Comisión la ostenta el ministro de la Presidencia y son convocados a sus reuniones los secretarios de Estado, los Subsecretarios de los Ministerios que tienen las facultades del artículo 15 de la Ley de Régimen Juridico de la Administración del Estado, los secretarios generales de los ministros adjuntos y el Jefe del Gabinete del presidente del Gobierno desde que se le atribuyó la categoria de subsecretario. La Secretaria de la Comisión corresponde al secretario general técnico de la Presidencia del Gobierno.

Debe añadirse que la Comisión es convocada formalmente, con independencia de las reuniones del Gobierno, por su presidente y sus acuerdos se hacen constar en acta repartida en la correspondiente sesión del Gobierno.

Además del mencionado acuerdo, se dictaron unas normas detallando el funcionamiento de la Comisión, completadas en noviembre del 77 por otras relativas al orden del día del Consejo de Ministros. Todas estas normas y la parte que queda en vigor de las instrucciones anteriormente reseñadas constituyen el marco regulador del funcionamiento actual del Gobierno que se describe a continuación.

\subsection{Reparto previo de documentos}

- los proyectos que van a ser elevados al Consejo de Ministros se reparten à través del Secretariado del Gobierno o directamente por el Ministerio proponente, enviándose duplicado, en el último supuesto, al Secretariado.

- el procedimiento usualmente utilizado para el envío es un télex dirigido al presidente del Gobierno, vicepresidentes y ministros, secretarios de Estado y subsecretario de Defensa.

- el plazo normal de reparto varía según se trate de disposiciones de carácter general, que deben circularse como minimo con ocho días de antelación a la reunión (jueves de 
la semana anterior a la de la sesión del Consejo, si ésta, como es habitual, tiene lugar el viernes), o de otros acuerdos, que se distribuyen con un margen de cuatro días. Otros temas, como las disposiciones que no tienen carácter general, los expedientes y los asuntos para información, no están sujetos a la obligación de reparto previo.

- por razones de urgencia, el plazo de reparto queda reducido a cuarenta y ocho horas previa petición del ministro interesado al ministro de la Presidencia. Usualmente el subsecretario respectivo anuncia esta intención en la Comisión General, distribuyendo el texto en la reunión. Con carácter excepcional, el presidente del Gobierno puede autorizar la deliberación de un asunto distribuido con menos de cuarenta y ocho horas o incluso sin haber sido objeto de reparto.

\subsection{Observaciones y contestaciones a LAS Mismas}

Las observaciones que los Ministerios juzgan oportuno formular a los textos, generalmente preparadas por las Secretarias Generales Técnicas (informes a los que se ha hecho alusión en el punto III. 1), son remitidas directamente al Ministerio proponente, con copia al Secretariado del Gobierno, con anterioridad a la celebración de la reunión de la Comisión General de Subsecretarios, si bien en ocasiones suelen entrecruzarse en la propia mesa de esta Comisión.

Análogo procedimiento se sigue respecto a la contestación de las observaciones, aunque debe destacarse la participación en su redacción de los Centros directivos en los que se inició la elaboración del proyecto.

\subsection{Convocatoria de las reuniones de la Comisión General de Subsecretarios y del Consejo de Ministros}

Las sesiones del Consejo de Ministros suelen tener lugar los viernes y su convocatoria se realiza mediante télex enviado por el ministro de la Presidencia a través del Secretariado del Gobierno. 
Respecto a la Comisión de Subsecretarios, el correspondiente télex de convocatoria es cursado por el secretario de Estado para la Administración Pública. Sus reuniones tienen lugar los miércoles, si bien inmediatamente después de su creación celebraba sesiones veinticuatro horas antes del Consejo de Ministros.

\subsection{FORMACIÓN DEL ORDEN DEL DÍA}

El orden del día que inicialmente se prevé para una reunión. del Gobierno se conoce con la denominación de "Indice negro".

Para su elaboración, los Ministerios remiten un indice parcial de asuntos que afectan a su Departamento, durante la mañana del lunes anterior a la reunión.

El secretario general técnico de la Presidencia somete al ministro el orden del día general, que suele ser repartido antes del término de ese mismo día.

Son causas que motivan la exclusión del orden del día:

- a) El no reparto o reparto fuera de plazo del proyecto de disposición.

b) La falta de aprobación previa de la Presidencia del Gobierno a que se refiere el artículo 130, 2, de la Ley de Procedimiento Administrativo cuando las disposiciones inciden en alguna de las materias enumeradas en el artículo 13, 7, de la Ley de Régimen Jurídico de la Administración del Estado.

c) La ausencia de conformidad de algunos de los Ministerios interesados en los supuestos de edisposiciones conjuntas".

d) La petición de retirada del asunto por el propio Ministerio proponente, si bien tales peticiones, que pueden obedecer a diversas razones de oportunidad, no suelen ser frecuentes.

Además de estas causas que juegan de una manera automática, suele ser motivo de no inclusión en el orden del día la ausencia de dictámenes o informes previos cuando es manifiesto su carácter preceptivo.

En más raras ocasiones alguna propuesta no es incluida en base a la falta de madurez en su elaboración puesta de relieve en la cantidad de observaciones que ha originado.

El «Indice negro" así formado es examinado por la Comisión de Subsecretarios. Aquellos asuntos informados favorablemente 
y por unanimidad por la Comisión, excepto las normas con rango de Ley, forman el denominado "Indice verde". Estos asuntos no son ya objeto de deliberación verbal por el Consejo de Ministros.

Con independencia de los asuntos que la Comisión estime, también por unanimidad, que deben quedar "sobre la mesa", es decir, ser objeto de reconsideración en una reunión posterior, los demás son elevados al Gobierno formando parte del llamado "Indice rojo". En consecuencia, se incluyen en este índice para ser objeto de especial atención y deliberación verbal del Consejo de Ministros:

a) Los proyectos en los que han existido discrepancias de opinión $\mathrm{y}$, en consecuencia, no ha recaído un acuerdo unánime.

b) Los proyectos de Decretos-leyes y anteproyectos de Leyes, en todo caso, y, excepcionalmente, otros asuntos favorablemente informados por unanimidad respecto a los que se estima deseable por su trascendencia, una deliberación formal del Gobierno.

c) Los asuntos que no figuran en el "Indice negro" y que, no obstante, se incluyen en el «Indice rojo» por razones de urgencia de acuerdo con los criterios antes apuntados.

Por lo demás, la estructura de estos Indices es la fijada en Instrucciones anteriores ya reseñadas.

\subsection{Reuniones}

Las normas antes reseñadas prestan alguna atención al procedimiento de las reuniones de la Comisión de Subsecretarios, si bien, dado su carácter adjetivo, no parece necesario describirlo. Las reuniones del Gobierno, por el contrario, no consta que hayan sido objeto de una regulación formal. No obstante, en la práctica, y desde hace años, el orden de intervención de los ministros viene determinado por el lugar que ocupan alrededor de la mesa del Consejo en función de la prelación establecida en el artículo $3 .^{\circ}$ de la Ley de Régimen Jurídico de la Administración del Estado y artículo 1. ${ }^{\circ}$ del Real Decreto 1558/1977, de 4 de julio. Alternativamente, el turno de intervenciones comienza en 
cada sesión por la derecha e-izquierda del presidente, lo que determina la estructura del orden del día.

\subsection{Actas}

Los acuerdos de la Comisión General de Subsecretarios se hacen constar en acta, que extiende el secretario general técnico de la Presidencia del Gobierno y que se reparte en la correspondiente sesión del Gobierno. Es un acta que se limita a relacionar los asuntos que se incluirán en el "Indice verde" -aquellos cuya aprobación se propone al Consejo de Ministros- y en el «Indice rojo" - los que se someten a la deliberación del Gobierno-, así como los que se estima deben quedar pendientes para volver a estudio de la Comisión.

El acta de las sesiones del Consejo de Ministros, que levanta el ministro de la Presidencia, tiene este mismo carácter formal. Antes de su aprobación se envía un borrador a los ministros para que formulen las observaciones que juzgen pertinentes, y una vez aprobada, se custodia en el Secretariado del Gobierno.

\subsection{Reflejo documental de los acuerdos del Gobierno}

La preparación del "dossier» correspondiente a los asuntos que se elevan al Gobierno la realiza cada Departamento, que cuida, en el supuesto de disposiciones de carácter general, del procedimiento de elaboración de las mismas. Finalizada la tramitación de las propuestas, se vierten en la documentación normalizada a la que ya nos hemos referido, sobre cuyos originales se certifica por el Secretario del Consejo el acuerdo recaído, devolviéndose al Ministerio proponente. La custodia, pues, de los expedientes relativos a las decisiones del Gobierno se realiza por los respectivos Departamentos, quedando solamente en la Secretaría del mismo una copia de la documentación normalizada.

\subsection{Consideraciones}

El funcionamiento del Gobierno tal como ha quedado descrito suscita en líneas generales unas consideraciones mínimas.

a) La organización de los trabajos del Gobierno no ha sido objeto ni lo es en la actualidad de una regulación completa a 
través de una sola norma. Como ya se ha puesto de relieve, desde el año 1958 se han sucedido una serie de circulares, instrucciones o acuerdos, que han ido dejando parcialmente en vigor parte de los anteriores -por ejemplo, se siguen utilizando, con algún retoque, los impresos normalizados aprobados en $1961-$ y que han centrado reiteradamente su atención en una serie de puntos, olvidando otros muchos. Así, por ejemplo, se ha insistido con extraordinaria frecuencia en los plazos de reparto o en la inclusión de asuntos en el orden del día por razones de urgencia, mientras que otros temas, como la celebración de las sesiones - el contenido de las actas, han sido cuidadosamente obviados.

En todo caso puede afirmarse que todas las instrucciones han respondido a una misma idea del trabajo colectivo del Gobierno, reflejo, en definitiva, del anterior sistema político. La nueva situación postconstitucional exige la redacción de unas nuevas instrucciones de funcionamiento que obviamente respondan al papel que al Gobierno le corresponde jugar dentro de la nueva concepción del Estado. Se trataría de atender no sólo a los aspectos puramente procedimentales, sino, sobre todo, a la regulación de las relaciones de los ministros entre sí, de éstos con el presidente y de éste con el Gobierno.

Pese al secreto o discreción que suele rodear al funcionamiento del Gobierno en la mayoría de los paises, parece conveniente que el Reglamento del funcionamiento del Gobierno, como ocurre, por ejemplo, en la República Federal Alemana, pudiera ser objeto de una disposición de carácter general del rango adecuado.

b) La innovación sustancial introducida últimamente en la mecánica del funcionamiento del Gobierno ha sido la constitución de la Comisión General de Subsecretarios.

Su actuación ha permitido descargar al Consejo de Ministros de la mayor parte de sus obligaciones de carácter administrativo y, por otro lado, su funcionamiento ha revestido un alto grado de eficacia en la medida en que ha logrado llegar a un acuerdo en un elevado porcentaje de los asuntos que se le han sometido.

Sin embargo, este modelo de funcionamiento parece entrañar ciertos riessgos. De una parte, no hay razón aparente que impida que el sistema se reproduzca en escalones inferiores de la 
Administración, de forma que, mẹdiante sucesivos aIndices verdes», se sustraiga la decisión real a órganos superiores a los que corresponde la responsabilidad formal de la decisión. De otra, el hecho de no haberse producido jurídicamente una desconcentración de funciones, no significa que no haya tenido lugar en la práctica, generando, aunque no fuera esa la finalidad pretendida, una duplicidad de centros de decisión política.

Pese a todo ello, la asistencia técnica que la Comisión presta al Gobierno la hace necesaria en la forma en que éste organiza hoy sus trabajos. No obstante, la previsible reconsideración de los niveles políticos del Gobierno - piénsese en la distorsión que ya supuso la creación de las Secretarias de Estado-, la regulación que se dé a las reuniones del Gobierno o de parte de sus miembros con independencia del Consejo de Ministros en sustitución de las actuales Comisiones Delegadas, la posibilidad de que se instituyan en nuestro ordenamiento Decretos no deliberados en Consejo que supondrían un auténtico cauce de desconcentración, la necesaria reestructuración de los órganos de apoyo de la Presidencia del Gobierno y la propia configuración que se haga de la figura del Subsecretario, van a ser factores que, entre otros, obligarán a reconsiderar en profundidad el futuro de la Comisión de Subsecretarios.

c) No resulta posible, ni siquiera útil, apuntar los criterios concretos a los que, desde un punto de vista instrumental, debería responder el Reglamento interno del Gobierno al que antes se ha hecho referencia. Hay que tener en cuenta que el correspondiente procedimiento de trabajo vendría condicionado por un conjunto de variables aún no decididas. La propia composición del Gobierno y su régimen de reuniones, la configuración de los servicios de apoyo directo al presidente del Gobierno, las características que reúna el programa del Gobierno y, sobre todo, la forma en que se plasme la titularidad de la potestad reglamentaria y el procedimiento para la elaboración de las normas generales, condicionan esencialmente los trabajos del Gobierno.

Sin embargo, sí existen algunos puntos minimos que parece pueden estar presentes en algunas hipótesis de trabajo.

- La necesaria existencia de un programa legislativo del Gobierno incidirá en la forma de elaboración del orden del día del 
Consejo de Ministros y en el sistema de plazos de reparto de textos y observaciones. El orden del dia responderá fundamentalmente al calendario en el que plasme el referido programa legislativo y no, como actualmente ocurre, a las iniciativas individuales de cada uno de los Departamentos.

- Cualquiera que sea la configuración de los órganos de apoyo al presidente en relación con los trabajos del Consejo, parece que su actuación debería diferir de la del resto de los Departamentos. Sus informes, a la vista del conjunto de observaciones; deberian reflejar, sobre todo, el grado de madurez del proyecto, con independencia de su participación preponderante en la perfección formal y puesta a punto final de la norma.

De otro lado, debería considerarse la oportunidad de que sean estos servicios los que gestionasen en exclusiva determinados proyectos, como los de Leyes y Decretos-leyes -así ocurre, por ejemplo, en el sistema francés-, y todas las hoy denominadas "disposicions conjuntas", respecto a las que la participación de la Presidencia es puramente formal pese a que se dicten con el refrendo del ministro de la Presidencia.

- Respecto a las propias reuniones del Gobierno, debería pensarse en la conveniencia de que asistiera a las mismas con ca: rácter permanente algún alto funcionario con la misión específica de asistir técnicamente al Consejo, y ello con independencia de los que puedan acompañar ocasionalmente a un ministro determinado para informes sobre un asunto especifico.

Es ésta una práctica que puede calificarse de usual en los distintos sistemas europeos. En Francia acude el secretario general del Gobierno, que, entre otras misiones, es el encargado de reflejar por escrito las deliberaciones. En Bélgica, el jefe del Gabinete del primer ministro. En la República Federal de Alemania, entre otros altos cargos, un secretario funcionario de la Cancilleria Federal, y en Inglaterra, el jefe del "Cabinet Secretariat":

De otro lado, las deliberaciones del Consejo, plasmen o no formalmente en un acta, quizá dẹan reflejarse con más detalle que en la actualidad. Se trataría de que se recogieran no sólo los acuerdos finales sino también, cuando así fuera exigido, las discrepancias de ọpinión a lo largo de la deliberación. Piénsese en la posibilidad de que se estableciera un limite temporal a la obli- 
gación de guardar secreto de las deliberaciones del Gobierno o en la existencia de Gobiernos de coalición. En algún país como Bélgica la práctica de levantar regularmente las actas se originó justamente por esta última circunstancia.

5. En la descripción del trabajo colectivo del Gobierno corresponde ahora hacer una referencia a la remisión de los proyectos de Ley a las Cortes y a la publicación de sus acuerdos en el «Boletín Oficial del Estado» (

El procedimiento 'de remisión de acuerdos del Gobierno a las Cortes y de publicación en el «BOE» de los actos y normas emanadas de las mismas, ha sido objeto de regulación recientemente a través de un Acuerdo del Consejo de Ministros de 24 de noviembre de 1978, cuya finalidad esencial ha sido cuidar de la autenticidad de los textos.

Los principios más destacables del contenido de este acuerdo son:

- la centralización a través del «ministro secretario del Gobierno" de las apuntadas relaciones.

- cierto énfasis en los requisitos formales de diligenciación.

- la atribución de la custodia material de los textos al Secretariado del Gobierno.

En definitiva, parece que el acuerdo supone un primer intento en la línea antes apuntada de acercar el procedimiento de elaboración de los proyectos de Leyes a los servicios directamente dependientes del presidente del Gobierno.

La última fase del procedimiento que se viene examinando la constituye la publicación de los acuerdos del Gobierno en el periódico oficial, que se encuentra regulada en el reglamento del "BOE" (aprobado por Decreto 1583/1960, de 10 de agosto, modificado parcialmente en sucesivas ocasiones).

Se trata del único punto de los abordados en este trabajo objeto de una disposición de carácter general.

Este Reglamento tiene un contenido más amplio que el procedimiento de inserción de disposiciones en cuanto que regula los distintos aspectos del Boletín Oficial del Estado como organismo autónomo adscrito a la Presidencia del Gobierno a través de su Secretaria General Técnica. 
Por lo que se refiere propiamente a la publicación de disposiciones y otros acuerdos del Gobierno se sigue una tramitación que se inicia en el Secretariado del Gobierno cuando son devueltas a los Ministerios las correspondientes propuestas diligenciadas. Cada Departamento vierte el texto aprobado en unos impresos normalizados en base a los cuales se prepara el ejemplar especial que, refrendado por el respectivo ministro, se somete a la firma del Rey.

Con independencia de que este procedimiento, que sufre sucesivas transcripciones y traslados, pudiera ser simplificado como consecuencia del correspondiente análisis de procesos, conviene resaltar que la responsabilidad de la orden de publicación radica básicamente en el Departamento proponente. A estos efectos, en el Secretariado del Gobierno y en el Boletín existe un fichero de firmas, debidamente autenticadas, que están facultadas para ordenar la inserción.

Parece claro que cuando se aborde la reglamentación de los trabajos del Gobierno será el momento de reconsiderar si el "insértese", es decir, el control de la autenticidad, debiera quedar reservado, como ocurre en Francia, a la Secretaría del Gobierno, de modo que ningún texto emanado del Gobierno se publicara sin que este órgano diera su conformidad.

6. La Secretaría del Gobierno, desde el punto de vista orgánico, es objeto de atención en dos artículos de la Ley de Régimen Jurídico de la Administración del Estado. El último párrafo del artículo $3 .^{\circ}$ establece que el presidente del Gobierno estará asistido por el ministro subsecretario de la Presidencia (hoy ministro de la Presidencia), al que corresponderá además la Secretaría del Consejo de Ministros. Por su parte, el artículo 12 alude al Secretariado del Gobierno, si bien con la denominación de Secretariado de las Comisiones Delegadas, atribuyéndole las funciones ya reseñadas, entre las que destaca la de "levantar el acta de los acuerdos adoptados y velar por su ejecución”.

De la simple lectura de estos dos preceptos pudiera entenderse erróneamente que, además del Ministro, asistiera a las reuniones del Gobierno el titular del Secretariado, ya que ambos órganos son objeto de un tratamiento legal aparentemente independiente. 
Estudios

La realidad, sin embargo, demuestra que solamente el ministro ha asistido a las reuniones del Consejo y que el Secretariado se ha configurado como un órgano de carácter técnico, encuadrado en la estructura de la Presidencia, con nivel orgánico no muy elevado y alejado jerárquicamente del ministro.

El ministro que ha desempeñado la Secretaría del Consejo de Ministros desde la Ley de Régimen Jurídico hasta nuestros días ha sido el ministro de la Presidencia, salvo el periodo en que fue atribuida al ministro de Información y Turismo por delegación de aquél en virtud de la Orden de 5 de octubre de 1967. Es el momento en que las funciones del ministro subsecretario de la Presidencia confluyen en el vicepresidente del Gobierno, por lo que el Decreto de la Jefatura del Estado 2346/1967, de 5 de octubre, autorizó dicha delegación.

Por su parte, el Secretariado del Gobierno constituye desde su creación una unidad de la Secretaría General Técnica de la Presidencia del Gobierno, si bien su nivel orgánico y dependencia directa han variado en los sucesivos Decretos de reorganización. Así, en el Decreto 245/1968, de 15 de febrero, se configuraba como una Sección de la Vicesecretaria General Técnica. En el año 1971, por Decreto 1446, de 11 de junio, alcanza el nivel de Subdirección General, que ha conservado hasta la actualidad. $\mathrm{Su}$ estructura vigente viene establecida por el Decreto 2761/1977, de 21 de octubre, y es la siguiente:

- Jefatura adjunta, con nivel de Servicio.

- Servicio de Asuntos del Consejo de Ministros.

- Servicio de Registro y Publicación de Disposiciones.

Debe advertirse que durante algún tiempo el Secretariado del Gobierno estuvo encuadrado en la hoy extinguida Dirección General de Relaciones Institucionales (Decreto 573/1974, de 7 de marzo). En esta época es el subsecretario de la Presidencia, cargo recién desdoblado del "ministro-subsecretario", el que en realidad coordina dicho Centro directivo con la Secretaría General Técnica, que habia conservado parte de las funciones de asistencia al Consejo.

$Y$ es que en la práctica el verdadero jefe del Secretariado del Gobierno, es decir, el que ejerce las funciones que en su dia 
atribuyó la Ley a este órgano, es el secretario general Técnico de la Presidencia, de quien dependen no sólo la oficina "Secretariado del Gobierno", básicamente dedicada a temas de tramitación y coordinación, sino también las demás unidades, como las de informes, que confluyen en la preparación de los trabajos del Gobierno.

La configuración del órgano encargado de la Secretaría del Gobierno difiere en los distintos sistemas europeos.

En Inglaterra, el «Cabinet Secretariat» forma parte del «Cabinet Office», que viene a ser el órgano de apoyo al primer ministro en cuanto jefe del equipo político. Su estructura no tiene carácter jerárquico, sino que la integran un conjunto de altos funcionarios con distinta categoría personal, y su procedimiento de trabajo no ha sido objeto de una norma específica.

El jefe del Gabinete del primer ministro es el que asume en Bélgica la Secretaría del Consejo de Ministros. Se trata de un órgano de confianza política y que por ello no está mayoritariamente formado por funcionarios permanentes.

En la República Federal de Alemania, bajo la autoridad del canciller, es el jefe de la Cancilleria Federal el que forma el orden del día y asiste a las reuniones del Gobierno. No existe una centralización de los trabajos previos a las sesiones, pero en contrapartida la tramitación de los expedientes del Gabinete se encuentra minuciosamente regulada.

El Secretariado General del Gobierno francés está configurado desde 1945 como un puesto superior permanente de la Administración, y participa activa y centralizadamente en la definición del contenido de la actuación gubernamental.

Los sistemas apuntados responden naturalmente al peculiar contexto político y administrativo de cada pais. En España la nueva configuración de la Secretaría del Gobierno vendrá condicionada por la propia composición de éste que la ley establezca, las relaciones que se perfilen entre presidente y ministros, $y$, sobre todo; la delimitación de la figura del presidente del Gobierno y del esquema orgánico general de la Presidencia del Gobierno. No obstante, parece posible adelantar algunas ideas generales sobre la organización y funciones de este órgano. 
Quizá convenga, en primer lugar, superar la confusión actualmente existente entre ministro y Ministerio de la Presidencia. Esta confusión es relativamente reciente en nuestra Administración, ya que arranca de la'Ley 1/1974, de 2 de enero, que crea el “Departamento de la Presidencia del Gobierno».

El conjunto de órganos de apoyo al presidente del Gobierno debe constituir la Presidencia del Gobierno, sin que exista inconveniente para que el titular de alguno o algunos de ellos tenga la categoria de ministro. Ahora bien, esto no debe llevar consigo su conversión en Departamento ministerial porque ello implicaría serios riesgos. De una parte, el presidente puede quedar en alguna medida desasistido, viéndose obligado a duplicar sus servicios de apoyo. De otra, y por lo que aquí nos interesa, determinados órganos de carácter general y con incidencias en el conjunto de la Administración, entre ellos la Secretaria del Gobierno, se verian disminuidos en su nivel y en las relaciones que deben mantener con el resto de los Departamentos.

Las notas que pudieran caracterizar a un futuro secretario general del Gobierno podrian ser:

a) Su dependencia directa del presidente del Gobierno.

b) Su permanencia y profesionalidad, a efectos de dotar de una cierta estabilidad y carácter técnico a los trabajos de apoyo al Consejo de Ministros. En este sentido podría incluso reservarse la titularidad del órgano a funcionarios de cualesquiera de las Administraciones Públicas.

c) Asistiria a las reuniones del Gobierno, con voz y sin voto, quedando sometido a la misma obligación de secreto que se imponga a los ministros.

d) No parece necesario que se le atribuyera expresamente un nivel orgánico o categoría personal determinada.

e) Sus funciones básicas podrian ser:

- partiendo del programa de actuación gubernamental, elaborar el calendario de pasos precisos para la aprobación de las más importantes disposiciones generales;

- vigilar e impulsar el cumplimiento de este calendario en sus distintas fases, estando presente en cada una de ellas; 
- formar el orden del día de las reuniones del Gobierno, elaborar las notas respecto a la madurez de los asuntos que se eleven al mismo y confeccionar las correspondientes actas;

- cuidar de la perfección formal de los textos y de su publicación en el diario oficial, así como de la remisión de los mismos a las Cortes y otros aspectos técnicos en las relaciones con éstas.

f) Su organización no debería estar excesivamente jerarquizada, sino que de la misma formarian parte expertos en las distintas áreas administrativas, sin que éstas tuvieran que coincidir necesariamente con las competencias de los Departamentos ministeriales. Además, se integrarian en su estructura los servicios administrativos necesarios, el Boletín Oficial del Estado y la oficina legislativa, que atendería fundamentalmente a la puesta al día de los textos legales y reglamentos en vigor.

\section{Conclusiones}

El tratamiento constitucional del Gobierno y de la Administración ha quedado reflejado en diversos articulos $\mathrm{y}$ hace difícil la determinación exacta del número y ámbito de las leyes precisas para su desarrollo.

El artículo 98, 1, alude indirectamente a una ley que establezca la composición del Gobierno, y el número 4 de este mismo precepto exige una ley para regular el Estatuto e incompatibilidades de sus miembros. De otro lado, la ley a que se refiere el artículo 103, 2, determinará la forma en que serán creados, regidos y coordinados los órganos de la Administración. Por último, y con independencia de otras remisiones legales a instituciones administrativas específicas, el artículo $149,1,18$, reserva a la competencia exclusiva del Estado las bases del régimen jurídico de las Administraciones Públicas.

Cualquiera que sea la opción que se siga, se entiende que la Ley de organización y funcionamiento del Gobierno -con apoyo más directo en el citado artículo 98,1 , o la parte de una ley con 
un ámbito más extenso, debería regular algunos de los puntos considerados en las páginas anteriores. En concreto, parece que deberia ser objeto de tratamiento legal lo siguiente:

1. Composición del Gobierno y de las Comisiones de Ministros.

2. Creación de la figura del secretario general del Gobierno, que podría ser nombrado por Real Decreto acordado en Consejo de Ministros entre funcionarios de cualesquiera de las Administraciones Públicas y que estaria sometido al mismo deber de secreto que los miembros del Gobierno. La ley establecería las líneas generales de sus atribuciones.

3. Asistencia regular a las reuniones del Gobierno de otras personas que no sean miembros del mismo, entre ellas el secretario general del Gobierno, asi como la asistencia esporádica de otros altos funcionarios para informar sobre cuestiones específicas.

4. Normas básicas del funcionamiento del Gobierno, entre ellas:

- convocatoria de las reuniones y formación del orden del dia, en ambos casos por el secretario general del Gobierno de acuerdo con las instrucciones del presidente;

- determinación del quórum de asistencia y de adopción de acuerdos;

- fijación de los límites del deber de secreto;

- autorización al Gobierno para dictar un Reglamento general de su funcionamiento.

Por su parte, el Reglamento que desarrolle estos preceptos de la ley debería establecer con minuciosidad las normas precisas para el buen orden de los trabajos del Gobierno y preparación de sus decisiones. Su ámbito, por tanto, se extenderia desde la formalización inicial de las propuestas hasta su publicación en el periódico oficial.

Madrid, noviembre de 1978. 


\section{NOTA BIBLIOGRAFICA}

No existe una bibliografía especifica sobre los métodos de trabajo del Gobierno. Algunas referencias, sin embargo, pueden encontrarse en obras más generales sobre la figura del primer ministro, el Gobierno y la coordinación de la política gubernamental. A estos efectos pueden citarse: La Presidenza del Consiglio dei ministri en alcuni Stati dell' Europa Occidentale ed ni Italia, PaOla Viviani, Milán, 1970; La coordination de la politique gouvernementale, volumen coléctivo bajo la dirección de Heinatch Stedentoff, París, 1976; Government in the Federal Republic of Germany, Nevil Johnson, Oxford, 1973; The reorganisation of Central Government, HMSO, 1970, Cmnd. 4506; The British Calbinet, J. P. Mackintosh, Londres, 1968; Cabinet Government, I. JENaInGs, Cambridge, 1969; The Management of Government, JoHN GARRET, Penguin Books, 1972; La Comédie du pouvoir, F. Grroud, 1977; La présidence du Conseil dans les institutions politiques de la France, J. MaRCEL, La Documentation Française, 1968; El Consejo de Ministros, A. Guaita, Madrid, 1967.

La aproximación a la realidad del funcionamiento interno del Gobierno debe hacerse a través del conocimiento directo de la práctica diaria de los diferentes sistemas. En los casos en que esta práctica se ha formalizado juridicamente y se ha hecho pública, el análisis de estas normas resulta obligado. En este sentido debe consultarse el dossier de la legislación francesa publicado por Francisco Santaolalla en el número 172 de Documentación Administrativa y los textos legales sobre el Gobierno de la República Federal Alemana traducidos por Miguel Angel Pérez de la Canal en el número 178 de Documentación Administrativa.

\section{NOT A}

Con posterioridad a la elaboración de esta Ponencia, en noviembre de 1978, se han producido algunas importantes vicisitudes que inciden en el contenido del trabajo. Dado que la publicación del mismo en Documentación Administrativa tiene lugar a los dos años de su redacción, parece conveniente reseñar brevemente las mús significativas.

1. Por Real Decreto $126 / 1980$, de 18 de enero, el ministro de la Presidencia quedó encargado de las relaciones del Gobierno con las Cortes, hasta el momento confiadas a un ministro adjunto sin Cartera. Fórmula ésta que puede suponer un primer paso en la línea señalada para un posible diseño de la Secretaria General del Gobierno. 
2. Suprimida la Secretaria de Estado para la Administración Pública y creada la Subsecretaría del Ministerio de la Presidencia en virtud del Real Decreto 1778/1980, de 13 de junio, hay que advertir que las menciones de aquel órgano contenidas en el trabajo deben entenderse referidas a dicha Subsecretaria.

3. El 5 de diciembre de 1979 se dictaron unas nuevas instrucciones para el funcionamiento de la Comisión General de Subsecretarios, que modifican las que en el texto de la Ponencia se recogen como vigentes, sin sustituirlas en su totalidad.

Las innovaciones que se introducen van básicamente dirigidas a obtener un mayor rigor en la elaboración previa de los textos dispositivos para evitar la frecuente constitución de grupos de trabajo en el seno de la propia Comisión que ultimen proyectos que llegan a la misma no suficientemente maduros y la necesidad de posibles sucesivas modificaciones de las disposiciones ya publicadas.

A este fin:

- se amplía en dos días la fecha de tramitación de proyectos de disposiciones de carácter general;

- se impide la inclusión en el orden del día de asuntos no aprobados en reuniones anteriores, salvo que sean objeto de nuevo reparto con inclusión de las observaciones acordadas y justificación de las rechazadas;

- se pone especial énfasis en que no se incluirán los proyectos en los que no hayan recaído los dictámenes, informes o aprobaciones que tengan carácter preceptivo;

- el ministro de la Presidencia puede excluir del orden del día o incluir sólo a título de información aquellos asuntos con un insuficiente grado de elaboración a la vista de las observaciones recibidas;

- respecto a los asuntos no incluidos en el orden del día, se adopta un criterio más restrictivo para su elevación al Consejo de $\mathrm{Mi}$ nistros y valoración de las razones de urgencia;

- las observaciones a los proyectos deben cursarse con mayor antelación, presumiéndose la conformidad si la disposición proyectada no es objetada dentro del plazo fijado. Con ello se pretende evitar la presentación de observaciones verbales a la vez que el Departamento proponente cuenta con tiempo suficiente para preparar la contestación; 
- por último, se prevén reglas más flexibles en relación con los anteproyectos de ley incluidos en el programa de desarrollo constitucional y con los anteproyectos de ley y normas reglamentarias cuyos textos revistan gran extensión.

4. El 28 de septiembre de 1979 el Gobierno acordó remitir a las Cortes Generales un proyecto de eLey Orgánica del Gobierno, de la Administración del Estado y de la Función Pública», publicado en el *Boletín Oficialı de las mismas, serie A, número 109-I, de 5 de febrero de 1980.

El proyecto aborda en esencia (capítulo V del título I, "Del funcionamiento del Consejo de Ministros:) los puntos que en las conclusiones del trabajo se estiman que deben ser objeto de tratamiento legal.

Dos cuestiones, sin embargo, parecen tratadas con un enfoque diferente al indicado en la Ponencia: el secretario del Gobierno, que deberá ser necesariamente un ministro y no el órgano más profesionalizado y estable que se propugnaba, y la regulación del funcionamiento del Gobierno, que plasmará en unas anormas internas», lo que parece apartarse de la idea de un Reglamento general.

De otro lado, el proyecto incide, a lo largo de su articulado, en otros temas que son objeto del contenido del estudio, entre ellos el procedimiento de elaboración de anteproyectos de ley y normas reglamentarias (en especial, artículo 35), la regulación de la potestad reglamentaria del Gobierno, que recoge la posibilidad de los decretos del presidente y del refrendo múltiple, y la nueva configuración de la Subsecretaria. 